\title{
Correction to: Meta-Analysis of Children's Learning Outcomes in Block-Based Programming Courses
}

Jen-I Chiu and Mengping Tsuei

\section{Correction to:}

Chapter "Meta-Analysis of Children's Learning Outcomes in Block-Based Programming Courses" in: C. Stephanidis et al. (Eds.): HCI International 2020 - Late Breaking Posters, CCIS 1294, https://doi.org/10.1007/978-3-030-60703-6_33

The originally published version of the chapter 33 contained a typesetting error in the Table 1. This has been corrected. 